\title{
Eigenvalue Intervals for Fractional Boundary Value Problems with Nonlinear Boundary Conditions
}

\author{
Meng Zhang*, Qiuping Li \\ Department of Mathematics, University of Jinan Quancheng Colllege, Penglai, P R China
}

\section{Email address:}

zhang123meng@163.com (Meng Zhang)

${ }^{*}$ Corresponding author

\section{To cite this article:}

Meng Zhang, Qiuping Li. Eigenvalue Intervals for Fractional Boundary Value Problems with Nonlinear Boundary Conditions. International Journal of Theoretical and Applied Mathematics. Vol. 3, No. 1, 2017, pp. 49-53. doi: 10.11648/j.ijtam.20170301.18

Received: October 29, 2016; Accepted: January 6, 2017; Published: January 18, 2017

\begin{abstract}
In presented paper, we study eigenvalue intervals for fractional boundary value problems with nonlinear boundary conditions. In this case, for the existence of at least one positive solution of the boundary value problem the new sufficient conditions are established. By means of example, the main results is illustrated. Finally, given comparsion obtained results with others.
\end{abstract}

Keywords: Fractional Differential Equation, Boundary Value Problem, Nonlinear Nonlocal Boundary Condition, Positive Solution, Fractional Green's Function, Guo-Krasnosel'skii Fixed Point Theorem

\section{Introduction}

Fractional differential equations have attracted great attention of mathematicians. It is caused both by the intensive development of the theory of fractional calculus itself and by the applications, see [1-4]. Recently, many scholars used techniques of nonlinear analysis (fixed-point theorems, Leray-Schauder theory, Adomian decomposition method, etc.) to deal with the existence of positive solutions of nonlinear boundary value problems of fractional differential equation, see [5-12].

$\mathrm{Xu}$ et al. [13] considered the existence of positive solutions for the following problem

$$
\begin{gathered}
D_{0^{+}}^{\alpha}(u(t))=f(u(t))=0,0<t<1, \\
u(0)=u(1)=u^{\prime}(0)=u^{\prime}(1)=0,
\end{gathered}
$$

where $3<\alpha \leq 4$ is a real number, $D_{0^{+}}^{\alpha}$ is the RiemannLiouville fractional derivative. By using the properties of the Green function, they gave some multiple positive solutions for singular and nonsingular boundary value problems, and also they gave uniqueness of solution for singular problem by means of Leray-Schauder nonlinear alternative, GuoKrasnosel'skii fixed point theorem on cones and a mixed monotone method.
Zhao et al. [14] studied the existence of positive solutions for the nonlinear fractional differential equation boundary value problem

$$
\begin{gathered}
D_{0^{+}}^{\alpha}(u(t))=\lambda f(u(t))=0,0<t<1, \\
u(0)+u^{\prime}(0)=0, u(1)+u^{\prime}(1)=0,
\end{gathered}
$$

where $1<\alpha \leq 2$ is a real number, $D_{0^{+}}^{\alpha}$ is the Caputo fractional deriva-tive, $\lambda>0$ and $f:[0,+\infty) \rightarrow[0,+\infty)$ is continuous. By the properties of the Green function and Guo-Krasnosel'skii fixed point theorem on cones, the eigenvalue intervals of the nonlinear fractional differential equation boundary value problem are considered, some sufficient conditions for the nonexistence and existence of at least one or two positive solutions for the boundary value problem are established.

Sun et al. [15] studied the existence of positive solutions for the nonlinear fractional differential equation boundary value problem

$$
\begin{gathered}
D_{0^{+}}^{\alpha}(u(t))=\lambda f(u(t))=0,0<t<1, \\
u(0)=u(1)=u^{\prime}(0)=u^{\prime}(1)=0
\end{gathered}
$$

where $3<\alpha \leq 4$ is a real number, $D_{0^{+}}^{\alpha}$ is the RiemannLiouville fractional derivative, $\lambda>0$ and $f:(0,+\infty) \rightarrow(0,+\infty)$ 
is continuous. By the properties of the Green function and Guo-Krasnosel'skii fixed point theorem on cones, the eigenvalue intervals of the nonlinear fractional differential equation boundary value problem are considered, some suff cient conditions for the nonexistence and existence of at least one or two positive solutions for the boundary value problem are established.

There also results on fractional boundary value problem with integral boundary conditions, such as Vong [16] studied positive solutions of the nonlocal boundary value problem for a class of singular fractional differential equations with an integral boundary condition,

$$
\begin{gathered}
{ }^{C} D_{0^{+}}^{\alpha}(u(t))+f(t, u(t))=0,0 \leq t \leq 1 \\
u^{\prime}(0)=\cdots=u^{(n-1)}(0)=0, u(1)=\int_{0}^{1} u(s) d \mu(s)
\end{gathered}
$$

where $n \geq 2, \alpha \in(n-1, n)$ and $\mu$ is a function of boundary variation. By the method of upper and lower solutions together with the Schauder fixed point theorem, the existence results for the boundary value problem are obtained.

Feng et al. [18] considered the existence of at least one positive solution of the fractional differential equation

$$
\begin{gathered}
D_{0^{+}}^{\alpha}(u(t))+f(u(t))=0,0<t<1, \\
u(0)=0, u(1)=H_{1}(\phi(u))+\int_{E} H_{2}(u(s)) d s,
\end{gathered}
$$

where $E \subset(0,1)$ is some measurable set, $1<\alpha \leq 2$ is a real number, $D_{0^{+}}^{\alpha}$ is the Riemann-Liouville fractional derivative, $u(1)=H_{1}(\phi(u))+\int_{E} H_{2}(u(s)) d s \quad$ represents a nonlinear nonlocal boundary condition. By imposing some relatively mild structural conditions on $f, H_{1}, H_{2}$ and $\varphi$, some sufficient conditions for the existence of at least one positive solution for the boundary value problem are established.

Motivated by the above works, we discuss the existence of at least one positive solution of the boundary value problem

$$
\begin{gathered}
D_{0^{+}}^{\alpha}(u(t))+\lambda f(u(t))=0,0<t<1, \\
u(0)=0, u(1)=H_{1}(\phi(u))+\int_{E} H_{2}(u(s)) d s
\end{gathered}
$$

where $E \subset(0,1)$ is some measurable set, $1<\alpha \leq 2$ is a real number, $D_{0^{+}}^{\alpha}$ is the Riemann-Liouville fractional derivative, $\lambda$ is a positive parameter and $f:(0,+\infty) \rightarrow(0,+\infty)$ is continuous, $\varphi$ in (2) is a linear functional having the form

$$
\left.\phi(u):=\int_{0}^{1} u(t)\right) d \theta(t)
$$

The rest of this paper is organized as follows. In section 2, we review some necessary preliminaries and new properties for Green's function. In section 3, we present the main results of this paper; also we give an illustrative example to support our new results.

\section{Preliminaries}

In this section, we give some lemmas to facilitate analysis of problem (1) and (2). And the background materials from fractional calculus theory can be found in [17].

Lemma 1 (following in [18]) Let $u, h \in C[0,1]$ and $1<\alpha \leq$ 2. The unique solution of problem

$$
\begin{gathered}
D_{0^{+}}^{\alpha}(u(t))+h(u(t))=0,0<t<1, \\
u(0)=0, u(1)=H_{1}(\phi(u))+\int_{E} H_{2}(u(s)) d s
\end{gathered}
$$

is

$$
u(t)=t^{\alpha-1}\left[H_{1}(\phi(u))+\int_{E} H_{2}(u(s)) d s\right]+\int_{0}^{1} G(t, s) h(s) d s,
$$

Where

$$
G(t, s)=\left\{\begin{array}{cl}
\frac{t^{\alpha-1}(1-s)^{\alpha-1}-(t-s)^{\alpha-1}}{\Gamma(\alpha)}, & 0<s \leq t<1, \\
\frac{t^{\alpha-1}(1-s)^{\alpha-1}}{\Gamma(\alpha)}, & 0<t \leq s<1 .
\end{array}\right.
$$

Here $G(t, s)$ is called the Green function of boundary value problem (4) and (5).

The following properties of the Green function different from [10] play important roles in this paper.

Lemma 2 The function $G(t, s)$ defined by (6) satisfies the following conditions:

(1) $G(t, s)>0$, for $t, s \in(0,1)$;

(2) There exists a positive constant $\gamma \in(0, \gamma(\mathrm{t}, \mathrm{s}))$ satisfies $(\alpha-1) \gamma \leq t^{\alpha-1}$ such that

$$
(\alpha-1) \gamma t^{\alpha-1}(1-s)^{\alpha-1} \leq \Gamma(\alpha) G(t, s) \leq t^{\alpha-1}(1-s)^{\alpha-1},
$$

where $t, s \in(0,1)$ and $\gamma(t, s)=\min \{s(1-t), t(1-s)\}$.

Proof. By the expression of $G(t, s)$, it is clear that $G(t, s)>$ 0 and $\Gamma(\alpha) G(t, s) \leq t^{-1}(1-s)^{-1}$ for $t, s \in(0,1)$.

When $s \leq t$, we have

$$
\begin{aligned}
\Gamma(\alpha) \mathrm{G}(\mathrm{t}, \mathrm{s}) & =t^{\alpha-1}(1-s)^{\alpha-1}-(t-s)^{\alpha-1} \\
& =(\alpha-1) \int_{t-s}^{t-t s} x^{\alpha-2} d x \\
& \geq(\alpha-1) t^{\alpha-2}(1-s)^{\alpha-2} s(1-t) \\
& \geq(\alpha-1) t^{\alpha-2}(1-s)^{\alpha-1} s(1-t)
\end{aligned}
$$

On the other hand, when $s \geq t$, we have

$$
\begin{aligned}
& \Gamma(\alpha) \mathrm{G}(\mathrm{t}, \mathrm{s})=t^{\alpha-1}(1-s)^{\alpha-1} \\
= & (\alpha-1) \int_{0}^{t-t s} x^{\alpha-2} d x
\end{aligned}
$$




$$
\begin{aligned}
& \geq(\alpha-1) t^{\alpha-2}(1-s)^{\alpha-2} t(1-s) \\
& \geq(\alpha-1) t^{\alpha-1}(1-s)^{\alpha-1} t(1-s) .
\end{aligned}
$$

Let $\gamma(t, s)=\min \{(t(1-s)), s(1-t)\}$, then we can find a constant $\gamma \in(0, \gamma(t, s))$ satisfies $(\alpha-1) \gamma \leq t^{-1}$ such that $\Gamma(\alpha) G(t, s) \geq(\alpha-1) \gamma t^{-1}(1-s)^{-1}$. The proof is complete.

If we set $q(t)=t^{-1}, k(s)=(1-s)^{-1}$, then $(\alpha-1) \gamma q(t) k(s) \leq \Gamma(\alpha) G(t, s) \leq q(t) k(s)$.

Lemma 3 (following in [19]) Let $X$ be a Banach space, and let $P \subset X$ be a cone in $X$. Assume $\Omega_{1}, \Omega_{2}$ are open subsets of $X$ with $0 \in \Omega_{1} \subset \Omega_{1} \subset \Omega_{2}$, and let $S: P \rightarrow P$ be a completely continuous operator such that, either

$\left(\mathrm{A}_{1}\right)\|S w\| \leq\|w\|, w \in P \cap \partial \Omega_{1},\|S w\| \geq\|w\|, w \in P \cap$ $\partial \boldsymbol{\Omega}_{2}$, or

$\left(\mathrm{A}_{2}\right)\|S w\| \geq\|w\|, w \in P \cap \partial \Omega_{1},\|S w\| \leq\|w\|, w \in P \cap$ $\partial \Omega_{2}$.

Then $S$ has a fixed point in $P \cap\left(\left(\bar{\Omega}_{2} \backslash \Omega_{1}\right)\right.$.

For convenience, we give some assumptions which will be used in the next section.

$\left(C_{1}\right): H_{1}:[0,+\infty] \rightarrow[0,+\infty]$ and $H_{2}:[0,+\infty) \rightarrow[0,+\infty)$ be real-valued, continuous functions.

$\left(C_{2}\right)$ : For $u \in C[0,1]$, there exists a constant $M_{1} \in[0,1]$ satisfies $|\phi(u)| \leq M_{1}\|u\|$.

$\left(C_{3}\right)$ : For each given $\varepsilon>0$, there are $M_{\varepsilon}>0$ and a constant $M_{2}$ such that $\left|H_{1}(x)-M_{2} x\right|<\varepsilon M_{2} x$, whenever $x>$ $M_{\varepsilon}$.

$\left(C_{4}\right)$ : There exists a function $F:[0,+\infty) \rightarrow[0,+\infty)$ satisfying $F(x) \leq M_{3} x$, for some $M_{3} \geq 0$, having the property that for each $\varepsilon>0$ given, there exists $M_{\varepsilon}>0$ such that $\left|H_{2}(x)-F(x)\right|<$ $\varepsilon F(x)$, whenever $x>M_{\varepsilon}$.

\section{Main Results}

In this section, we will establish the existence of positive solution for boundary value problem (1) and (2) by fixed point theorem in [19]. As an application, an example is given to illustrate the main results.

Lemma4. Let Banach space $E=C[0,1]$ be endowed with the norm $\|u\|=\sup |u(t)|$. Define the cone $P \subset E$ by $P=\{u \in E$ : $u(t) \geq \gamma(\alpha-1)\|u\|, t \in[0,1]$ and the operator $A_{\lambda}: P \rightarrow E$ by

$$
\left(A_{\lambda} u\right)(t)=t^{\alpha-1}\left[H_{1}(\phi(u))+\int_{E} H_{2}(u(s)) d s\right]+\lambda \int_{0}^{1} G(t, s) f(u) d s
$$

Then, $A_{\lambda}: P \rightarrow P$.

Proof. By lemma 2.4 (see[19]), if $u \in P$, we have

$$
\begin{aligned}
& \left\|\left(A_{\lambda} u\right)(t)\right\|=\sup \left|t^{\alpha-1}\left[H_{1}(\phi(u))+\int_{E} H_{2}(u(s)) d s\right]+\lambda \int_{0}^{1} G(t, s) f(u) d s\right| \\
& \leq\left[H_{1}(\phi(u))+\int_{E} H_{2}(u(s)) d s\right]+\frac{\lambda}{\Gamma(\alpha)} \int_{0}^{1} q(t) k(s) f(u) d s .
\end{aligned}
$$

On the other hand,

$$
\begin{gathered}
\left(A_{\lambda} u\right)(t)=t^{\alpha-1}\left[H_{1}(\phi(u))+\int_{E} H_{2}(u(s)) d s\right]+\lambda \int_{0}^{1} G(t, s) f(u) d s \\
\geq t^{\alpha-1}\left[H_{1}(\phi(u))+\int_{E} H_{2}(u(s)) d s\right]+\frac{\lambda(\alpha-1) \gamma}{\Gamma(\alpha)} \int_{0}^{1} q(t) k(s) f(u) d s \\
\geq \gamma(\alpha-1)\left\|\left(A_{\lambda} u\right)(t)\right\| .
\end{gathered}
$$

Thus, $A_{\lambda}: P \rightarrow P$.

Lemma 5 The operator $A_{\lambda}$ in lemma 4 is completely continuous.

The proof of this lemma is similar to lemma 3.1 in [18], we omit it.

For convenience, we denote

$$
\begin{gathered}
M_{4}=\frac{1}{\Gamma(\alpha)} \int_{0}^{1} k(s) d s, M_{5}=\frac{(\alpha-1)^{2} \gamma^{2}}{\Gamma(\alpha)} \int_{0}^{1} k(s) d s, \\
F_{0}=\lim _{u \rightarrow 0^{+}} \sup \frac{f(u)}{u}, f_{\infty}=\lim _{u \rightarrow \infty} \sup \frac{f(u)}{u} .
\end{gathered}
$$

Theorem1 Assume $\left(C_{1}\right)-\left(C_{4}\right)$ hold, if there exists $\mathrm{s}$ $l \in(0,1)$ such that

$q(l) f_{\infty}(\alpha-1)^{2} \gamma^{2}\left(1-M_{1} M_{2}-M_{3} m(E)\right)>F_{0}$, then for each

$$
\lambda \in\left(\frac{1}{q(l) f_{\infty} M_{5}}, \frac{1-M_{1} M_{2}-M_{3} m(E)}{F_{0} M_{4}}\right),
$$

the boundary value problem (1) and (2) has at least one positive solution. Here we impose $\left(q(l) f_{\infty} M_{5}\right)^{-1}=0$, if $f_{\infty}=+\infty$ and $\left(F_{0} M_{4}\right)^{-1}=\infty$, if $F_{0}=0$.

Proof. Let $\lambda$ be in (8), we can find a very small $\varepsilon$ satisfy

$$
\frac{1}{q(l)\left(f_{\infty}-\varepsilon\right) M_{5}} \leq \lambda \leq \frac{1-M_{1} M_{2}-M_{3} m(E)}{\left(F_{0}+\varepsilon\right) M_{4}} .
$$

By the definition of $F_{0}=0$, there exists $r_{1}>0$ such that

$$
f_{u} \leq\left(F_{0}+\varepsilon\right) u, 0<u<r_{1} .
$$

So, if $u \in P$ with $\|u\|=r_{1}$, then by (9) and (10), we have

$\left\|\left(A_{\lambda} u\right)(t)\right\| \leq\left[H_{1}(\phi(u))+\int_{E} H_{2}(u(s)) d s\right]+\frac{\lambda}{\Gamma(\alpha)} \int_{0}^{1} q(t) k(s) f(u) d s+$ $\left.\leq\left|H_{1}(\phi(u))-M_{2}(\phi(u))\right|+\left|M_{2}(\phi(u))\right|+\int_{E}\left|H_{2}(u)-F(u)\right| d s\right]+\int_{E}|F(u)| d s$

$$
\frac{\lambda}{\Gamma(\alpha)} \int_{0}^{1} k(s)\left(F_{0}+\varepsilon\right) r_{1} d s
$$

$\leq M_{2} \varepsilon \phi(u)+M_{1} M_{2}\|u\|+(1+\varepsilon) M_{3} m(E)\|u\|+\lambda\left(F_{0}+\varepsilon\right) M_{4}\|u\|$

$\leq\left(M_{1} M_{2}(1+\varepsilon)+(1+\varepsilon) M_{3} m(E)+\lambda\left(F_{0}+\varepsilon\right) M_{4}\right)\|u\| \leq\|u\|$.

Hence, if we choose $\Omega_{1}=\left\{u \in E:\|u\|<r_{1}\right\}$, then 


$$
\left\|A_{\lambda} u\right\| \leq\|u\|, u \in P \bigcap \partial \Omega_{1} .
$$

From the definition of $f_{\infty}$, we can find $r_{3}>0$ such that

$$
f_{u} \geq\left(f_{\infty}-\varepsilon\right) u \text {, for } u \geq r_{3} .
$$

So, if $u \in P$ with $\|u\|=r_{2}=\max \left\{2 r_{1}, r_{3}\right\}$, then by (9) and (12), we have

$$
\begin{aligned}
\left\|\left(A_{\lambda} u\right)(t)\right\| & \geq\left(A_{\lambda} u\right)(l) \geq \lambda \int_{0}^{1} G(l, s) f(u(s)) d s \\
& \geq \frac{\lambda q(l)(\alpha-1) \gamma}{\Gamma(\alpha)} \int_{0}^{1} k(s) f(u(s)) d s \\
& \geq \frac{\lambda q(l)(\alpha-1)^{2} \gamma^{2}}{\Gamma(\alpha)} \int_{0}^{1} k(s)\left(f_{\infty}-\varepsilon\right)\|u\| d s \\
& =\lambda q(l) M_{5}\left(f_{\infty}-\varepsilon\right)\|u\| \geq\|u\| .
\end{aligned}
$$

Thus, if we set $\Omega_{2}=\left\{u \in E:\|u\|<r_{2}\right\}$, then

$$
\left\|A_{\lambda} u\right\| \geq\|u\|, u \in P \cap \partial \Omega_{2} .
$$

Now, from (11), (13) and Lemma 4, we guarantee that $A_{\lambda}$ has a fix point $u \in P \cap\left(\left(\bar{\Omega}_{2} \backslash \Omega_{1}\right)\right.$ with $r_{1} \leq\|u\| \leq r_{2}$, and clearly $u$ is a positive solution of (1) and (2). The proof is complete.

Now, we will present an example to illustrate the main result.

Example Let $f(u)=u^{a}, H_{1}(x)=x e^{x}+x, H_{2}(x)=3 u+u^{\frac{1}{2}}$, $\phi(u)=\frac{u}{2}$, consider the boundary value problem

$$
\begin{gathered}
D_{0^{+}}^{\frac{3}{2}}(u(t))+\lambda u^{a}=0,0<t<1 \\
u(0)=0, u(1)=\frac{u}{3} e^{-\frac{u}{3}}+\frac{u}{3}+\int_{\left[\frac{1}{10}, \frac{1}{5}\right]}\left(3 u+u^{\frac{1}{2}}\right) d s .
\end{gathered}
$$

It is easy to see $F_{0}=0, f_{\infty}=+\infty, m(E)=\frac{1}{10}$ and

$$
q(l) f_{\infty}(\alpha-1)^{2} \gamma^{2}\left(1-M_{1} M_{2}-M_{3} m(E)\right)>F_{0}
$$

Thus, by Theorem 1, the boundary value problem (14) and (15) has a positive solution for each $\lambda \in(0,+\infty)$.

\section{Conclusion}

In this work by means of preliminaries lemma1, 2, 3, 4, 5 proves the Theorem1, which is established the existence of positive solution for boundary value problem (1), (2) with fixed point theorem in [19]. This is a new sufficient conditions for the existence of at least one positive solution for the boundary value problem of (1), (2). which in case of $\lambda=1$, for boundary value problem (1), (2) incuding the result of [18].

\section{References}

[1] K. S. Miller, B. Ross, An Introduction to the Fractional Calculus and Fractional Differential Equation, John Wiley, New York, 1993.

[2] K. B. Oldham, J. Spanier, The Fractional Calculus, Academic Press, New York, 1974.

[3] I. Podlubny, Fractional Differential Equations, Academic Press, New York / Lindo n /Toronto, 1999.

[4] S. G. Samko, A. A. Kilbas, O. I. Marichev, Fractional Integral and Derivative. Theory and Applications, Gordon and Breach, Switzerland, 1993.

[5] D. Delbosco, L. Rodino, Existence and uniqueness for a nonlinear fractional differential equation, J. Math. Anal. Appl. 204 (1996) 609-625.

[6] Y. Zhao, S. Sun, Z. Han, Q. Li, Positive solutions to boundary value problems of nonlinear fractional differential equations, Abs. Appl. Anal. 2011 (2011) Article ID 390543, 1-16.

[7] Jiang, W. Eigenvalue interval for multi-point boundary value problems of fractional differential equations. Appl. Math. Comput. 219 (2013)4570-4575.

[8] Anguraj, A, Karthikeyan, P, Rivero, M, Trujillo, JJ, On new existence results for fractional integro-differential equations with impulsive and integral conditions. Comput. Math. Appl. 66 (2014), 2587-2594.

[9] D. Jiang, C. Yuan, The positive properties of the Green function for Dirichlet type boundary value problems of nonlinear fractional differential equations and its application, Nonlinear Anal. TMA 72 (2010) 710-719.

[10] Z. Bai, H. Lü, Positive solutions for boundary value problem of nonlinear fractional differential equation, J. Math. Anal. Appl. 311 (2005) 495-505.

[11] S. Zhang, Positive solutions for boundary value problems of nonlinear fractional differential equations, Electron. J. Differ. Equ. 36 (2006) 1-12.

[12] Y. Zhao, S. Sun, Z. Han, Q. Li, The existence of multiple positive solutions for boundary value problems of nonlinear fractional differential equations, Commun. Nonlinear Sci. Numer. Simul. 16 (2011) 2086-2097.

[13] X. Xu, D. Jiang, C. Yuan, Multiple positive solutions for the boundary value problem of a nonlinear fractional differential equation, Nonlinear Anal. 71 (2009) 4676-4688.

[14] Y. Zhao, S. Sun, Z. Han, M. Zhang, Positive solutions for boundary value problems of nonlinear fractional differential equations, Appl. Math. Comput. 217 (2011) 6950-6958.

[15] S. Sun, Y. Zhao, Z. Han, J. liu, Eigenvalue problem for a class of nonlinear fractional differential equations, Ann. Funct. Anal. 4 (2013) 25C39. 
[16] Vong, S, Positive solutions of singular fractional differential equations with integral boundary conditions. Math. Comput. Model. 57 (2013) 1053-1059.

[17] A. A. Kilbas, H. H. Srivastava, J. J. Trujillo, Theory and Applications of Fractional Differential Equations, Elsevier Science B. V., Amsterdam, 2006.
W. Feng, S. Sun, X. Li, M. Xu, Positive solutions to fractional boundary value problems with nonlinear boundary conditions. Boundary Value Problems 2014: 225, doi: 10.1186/s13661-014-0225-0.

[18] M. A. Krasnoselskii, Positive solution of operator equation, Noordhoff Groningen, 1964. 